and sister aged 25, the mother, and father were clinically normal and had normal electrical studies and MRIs, but their arylsulfatase activity was reduced. Allele-specific amplification confirmed the pseudodeficiency (PD) allele, with no clinical dysfunction, in the mother and her 2 unaffected children. The mother was a PD/MLD compound heterozygote since she was an obligate MLD carrier. The father was a normal (N/MLD) heterozygote. The family was unique in the unusual occurrence of MLD in consecutive generations in the absence of consanguinity. (Francis GS et al. Metachromatic leukodystrophy: multiple nonfunctional and pseudodeficiency alleles in a pedigree: problems with diagnosis and counseling. Ann Neurol Aug 1993;34:212-218). (Respond: Dr Francis, Department of Neurology, Montreal Neurologic Institute, McGill University, 3801 University Street, Montreal, Quebec, Canada H3A 2B4).

COMMENT. Three phenotypes of MLD are recognized based on age of onset: late infantile (0-2 years), juvenile (3-16 years), and adult. The time of onset is determined by the amount of residual arylsulfatase A activity, those retaining some activity having a delayed onset of the disease. A variety of allelic mutations has been identified, including a pseudodeficiency allele with no clinical dysfunction. Cloning of the enzyme gene and genotype identification at the molecular level for the PD and MLD mutations allow appropriate genetic counselling for the families. With these advances in diagnosis, the authors conclude that unnecessary testing of spouses and prenatal screening of pregnancies can be avoided.

\title{
MRI NEUROANATOMY OF RETT SYNDROME
}

The neuroanatomy of 11 females with Rett syndrome (RS) and 15 control subjects investigated in vivo with quantitative MRI is reported from Johns Hopkins University, Baltimore, MD. Compared to age- and gender-matched controls, RS patients had 1) significantly reduced cerebral volume; 2) reduction in brain tissue volumes, especially affecting gray matter; 3) regional variation in cortical gray matter, frontal regions showing the largest decrease; 4) volume reduction of caudate nucleus and midbrain; and 5) increased CSF volume. There was no evidence of an ongoing degenerative process in these patients. (Reiss AL et al. Neuroanatomy of Rett syndrome: a volumetric imaging study. Ann Neurol Aug 1993;34:227-234). (Respond: Dr Reiss, Behavioral Genetics and Neuroimaging, Kennedy Kreiger Institute, 707 North Broadway, Baltimore, MD 21205).

COMMENT. These findings are consistent with one previous neuropathological report, whereas cerebellar pathology was emphasized in another autopsy report of 5 patients (Oldfors A et al. Pediatr Neurol 1990;ㅁ:310). See Progress in Pediatric Neurology, PNB Publ, 1991. 